This item was submitted to Loughborough's Research Repository by the author.

Items in Figshare are protected by copyright, with all rights reserved, unless otherwise indicated.

\title{
Driving performance and driver discomfort in an elevated and standard driving position during a driving simulation
}

PLEASE CITE THE PUBLISHED VERSION

http://dx.doi.org/10.1016/j.apergo.2015.01.003

\section{PUBLISHER}

(C) Elsevier

\section{VERSION}

AM (Accepted Manuscript)

\section{PUBLISHER STATEMENT}

This work is made available according to the conditions of the Creative Commons Attribution-NonCommercialNoDerivatives 4.0 International (CC BY-NC-ND 4.0) licence. Full details of this licence are available at: https://creativecommons.org/licenses/by-nc-nd/4.0/

\section{LICENCE}

CC BY-NC-ND 4.0

\section{REPOSITORY RECORD}

Smith, Jordan, Neil J. Mansfield, Diane E. Gyi, Mark Pagett, and Bob Bateman. 2019. "Driving Performance and Driver Discomfort in an Elevated and Standard Driving Position During a Driving Simulation". figshare. https://hdl.handle.net/2134/17049. 
Applied Ergonomics

Volume 49, July 2015, Pages 25-33

http://dx.doi.org/10.1016/j.apergo.2015.01.003

\title{
Driving Performance and Driver Discomfort in an Elevated and Standard Driving
}

\section{Position During a Driving Simulation}

Jordan Smith ${ }^{\star 1,2}$, Neil Mansfield ${ }^{1}$, Diane Gyi ${ }^{1}$, Mark Pagett ${ }^{2}$ and Bob Bateman ${ }^{2}$

\author{
Loughborough Design School \\ Loughborough University \\ Loughborough \\ Leicestershire, LE11 3TU \\ UK
Nissan Technical Centre Europe
Cranfield Technology Park \\ Cranfield \\ Moulsoe Road \\ Bedfordshire, MK43 ODB \\ UK \\ *corresponding author \\ j.smith2@lboro.ac.uk \\ +441509226934
}




\begin{abstract}
The primary purposes of a vehicle driver's seat, is to allow them to complete the driving task comfortably and safely. Within each class of vehicle (e.g. passenger, commercial, industrial, agricultural), there is an expected driving position to which a vehicle cabin is designed. This paper reports a study that compares two driving positions, in relation to Light Commercial Vehicles (LCVs), in terms of driver performance and driver discomfort. In the 'elevated' driving position, the seat is higher than usually used in road vehicles; this is compared to a standard driving position replicating the layout for a commercially available vehicle. It is shown that for a sample of 12 drivers, the elevated position did not, in general, show more discomfort than the standard position over a 60 minutes driving simulation, although discomfort increased with duration. There were no adverse effects shown for emergency stop reaction time or for driver headway for the elevated posture compared to the standard posture. The only body part that showed greater discomfort for the elevated posture compared to the standard posture was the right ankle. A second experiment confirmed that for 12 subjects, a higher pedal stiffness eliminated the ankle discomfort problem.
\end{abstract}

\title{
Highlights
}

- An elevated driving posture does not adversely affect comfort

- An elevated driving posture does not adversely affect driver performance

- Accelerator pedal stiffness is associated with ankle discomfort

\section{Keywords}

Driver comfort, driver posture, driving performance 


\section{Introduction}

The design of vehicles (e.g. rail vehicles, trams, buses, cars, delivery vehicles, vans) for city use requires a balance between the benefits of being light and compact, and the benefits of having a large load capacity. Lightness and compactness can increase fuel economy and manoeuvrability. If it is possible to shorten the overall space required to accommodate the driver, the vehicle could benefit from a more compact design. Most current vehicle designs require the driver to sit in a low seat with a semi-recumbent posture with legs extended towards the front of the vehicle. If the height of the seat is increased the driving posture can be altered such that the feet are positioned further back, thus reducing the need for space in front of the driver. Further advantages of this elevated posture include improved ingress and egress for drivers and/or passengers, and improved visibility. Whilst some vehicles use an elevated driving posture, there is little evidence to determine the suitability of this posture for comfort and control of the vehicle.

Rebiffe (1969) explored the posture and position of the driver to best fit the requirements of the driving task and was able to propose theoretical joint angles of the body for comfort and correct posture. Porter and Gyi (1998) augmented this theoretical framework with observed driving postures. New guidelines for optimum postural comfort were developed regarding body angles and inter relationships between adjacent joint angles. It was noted that even with theoretically optimal postures, not all people will be comfortable with the whole range of adjustment practically achievable with production vehicles.

Postural assessment alone is insufficient to determine the overall comfort of a vehicle seat occupant. Mansfield et al. $(2005,2014)$ identified factors affecting discomfort that can include the seat shape itself, the fit of this to the occupant, the materials, the thermal environment, exposure to whole body vibration (WBV), opportunity for changing posture and the length of time sitting in the same seated position. These factors can be broken down through numerical analysis of subjective discomfort ratings in order to predict discomfort under different conditions. During driving, drivers will be exposed to vibrations from the road surface. Griffin et al. (1982) described how vibration, in combination with a 
seated posture increases the level of discomfort, especially during journeys of long duration. In real driving environments the vibration at the seat surface and backrest are transmitted through the body and interact with vibration from the steering wheel and pedals to form the sensation of vibration, which can lead to discomfort with increased vibration dose. El Falou et al. (2002) tested sensations of fatigue for two seat conditions, 'uncomfortable' and 'comfortable', and found that for both experimental conditions discomfort of the spine and back increased significantly over the 150-minute trial. Drivers are required to maintain vigilance over long periods of time during which they are exposed to wholebody vibration and can become fatigued. Studies of long-term discomfort have shown gradual increases in discomfort over time, irrespective of how well designed the seat might be. Whilst it is known that vibration accelerates the onset of discomfort it is currently unclear whether fatigue is also reflected in driving performance or vigilance.

One method of assessing driver performance is to use the measure of 'headway', defined as the distance from a lead car to a following car. Driving manuals and learner guidance often point towards a 2-second headway as being the ideal minimum safe distance (Department for Transport, 2007) when driving in normal conditions e.g. good visibility, dry road conditions. However, in reality the headway allowed by a driver can be influenced by the traffic density, the characteristics of the driver (Jonah, 1996), the perceived ability to judge physical situations better than other drivers (van Winsum, 1996), as well as the physical capabilities of the vehicle and circumstantial factors surrounding the journey e.g. routine vs. urgent journey. In some cases it is not possible to maintain a preferred headway if the space becomes occupied by other drivers changing lanes, therefore in practice the headway selected by 'safe' drivers is often less than 2 seconds (Rudin-Brown et al., 2004, SimonsMorton et al., 2005). With both of these studies classifying 'risky' driving as headways of $<1.5$ seconds, a 'time headway' of $\geq 1.5$ seconds in normal driving conditions (replicated in the driving simulation) was considered as safe for this study.

A second method of assessing driver performance is reaction time. The reaction time (RT) has important consequences for the design of safe roads and vehicles, and is predicted to be effective in establishing whether a posture change (the higher hip point) changes the time taken to move between 
the accelerator and brake pedal. Green (2000) conducted an analysis of driver perception-brake times and found that previous study results vary greatly because investigators have used many different signals, responses and testing conditions. Green continued that when fully aware of the time and location of the brake signal, drivers could move their foot from the accelerator (A) to the brake (B) pedal in approximately $0.70-0.75$ seconds. Green also noted that times can be affected by driver age, gender, cognitive load and the urgency of the driving situation. Engström et al. (2010) explored the effects of working memory load and repeated scenario exposure on emergency braking performance. A driving simulation was set-up whereby a lead car would pull in front of the driver at a predetermined time in the simulation, accelerate to headway of 1.5 seconds and then suddenly brake. The study decomposed the reaction time to the time taken to release the A pedal and then the time taken to switch the foot from the A pedal to the B pedal. The results indicated, firstly, that there was no effect of working memory load on accelerator-brake reaction time, indicating that this method of measurement is consistent. Secondly, the accelerator-brake reaction times were between 0.6-0.8 seconds. Decomposing the reaction time to discover the accelerator-brake time requires accurate data collection and lends itself to a high fidelity driving simulation. Whilst driver performance under 'normal' driving conditions is relatively well understood, the interaction of driver posture with performance is unknown.

This paper considers a study comparing a current production vehicle set up (the standard posture) with a seat height of $375 \mathrm{~mm}$ with a new specification driving position with an elevated seat height of $>400 \mathrm{~mm}$, the current maximum production hip point. It was hypothesised that the elevated driving posture would be no worse than the current vehicle in terms of comfort or performance. The reasoning for this is that the biomechanics of the elevated posture opens up the body angles (hip angle; knee angle) and moves them closer to their neutral and thus more comfortable position. Potential weight savings, benefits for visibility and ingress/egress were not investigated in this study. The current production vehicle is categorised as a 'small Light Commercial Vehicle (LCV)' and was set up with the production seat and corresponding adjustment slide, a standard pedal arrangement for the automotive sector and a standard steering system. Assessing comfort in this new elevated driving position allows this study to explore the range of optimum driving positions with an elevated seat height, with reference to the pedals and the steering wheel. This comparative experimental study 
used a motion platform and driving simulator to study discomfort ratings as well as fatigue effects, using self-selected headway and reaction time.

\section{Methods}

\subsection{Sampling}

12 participants, 6 males and 6 females were recruited from the population of staff and students at Loughborough University to take part in the trials. The criteria for recruitment were that each participant held a full UK driving license, had at least 1 full year of driving experience and was between the ages of $18-65$. The age range that was recruited was $20-60$ years. The Loughborough University Ethical Advisory Committee (LUEAC) approved the study.

\subsection{Equipment}

Two driving rigs were constructed for the study: the first represented a driving position (standard posture) from a small LCV production vehicle with conventional pedal and steering operations and actual seat slide adjustment range, as illustrated in Figure 1. The driving rig was set up for Automatic Transmission with just the accelerator (A) and brake (B) pedals and a fixed steering wheel position. The seat height for this small LCV production vehicle was $375 \mathrm{~mm}$ in $\mathrm{Z}$, with the distance to the pedals from the front edge of the seat ranging from $245 \mathrm{~mm}$ (foremost position) to $475 \mathrm{~mm}$ (rearmost position) in $X$. The seat base length was $460 \mathrm{~mm}$ in $X$, from the front edge of the seat cushion to the point at which the cushion meets the backrest. The standard posture rig was built using carry over parts from the vehicle including the seat, steering wheel and pedal set with replicated pedal stiffness. 


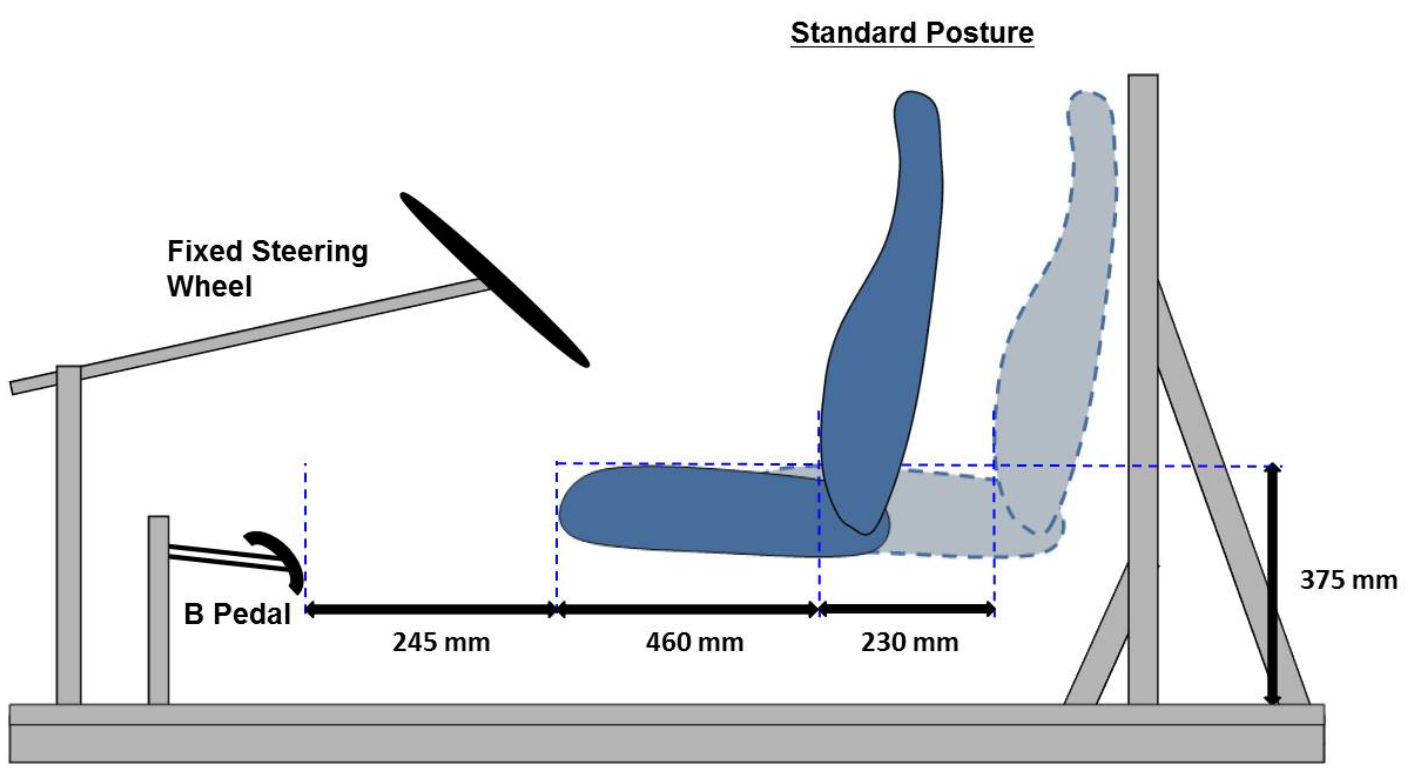

Figure 1. Image of seat, pedals and steering wheel used for the 'standard posture' condition.

The second rig was designed to accommodate an elevated seat height and a shorter seat base length, with adjustability in both the seat height and the distance from the pedals, as illustrated in Figure 2. The seat height had an adjustment range from $400 \mathrm{~mm}-800 \mathrm{~mm}$ in $Z$. The seat base length was $350 \mathrm{~mm}$ in $\mathrm{X}$, from the front of the seat cushion to the point at which the cushion meets the backrest. The distance from the seat to the pedals had an adjustment range between $450 \mathrm{~mm}-850 \mathrm{~mm}$ in X. The steering wheel had nominally unlimited adjustment built in with the capability to change the height of the wheel from the floor in $Z$, the distance from the driver in $X$ and the angle of the wheel itself. However, the steering wheel position was not a main focus of this study. The elevated posture rig was created using the same production steering wheel, however the seat was fabricated from two existing production seats and the pedal levers were fabricated from available materials with standardised pedal pads. Resistance was added to the pedals to give drivers tactile feedback when operating the pedals, comparable to real driving. 


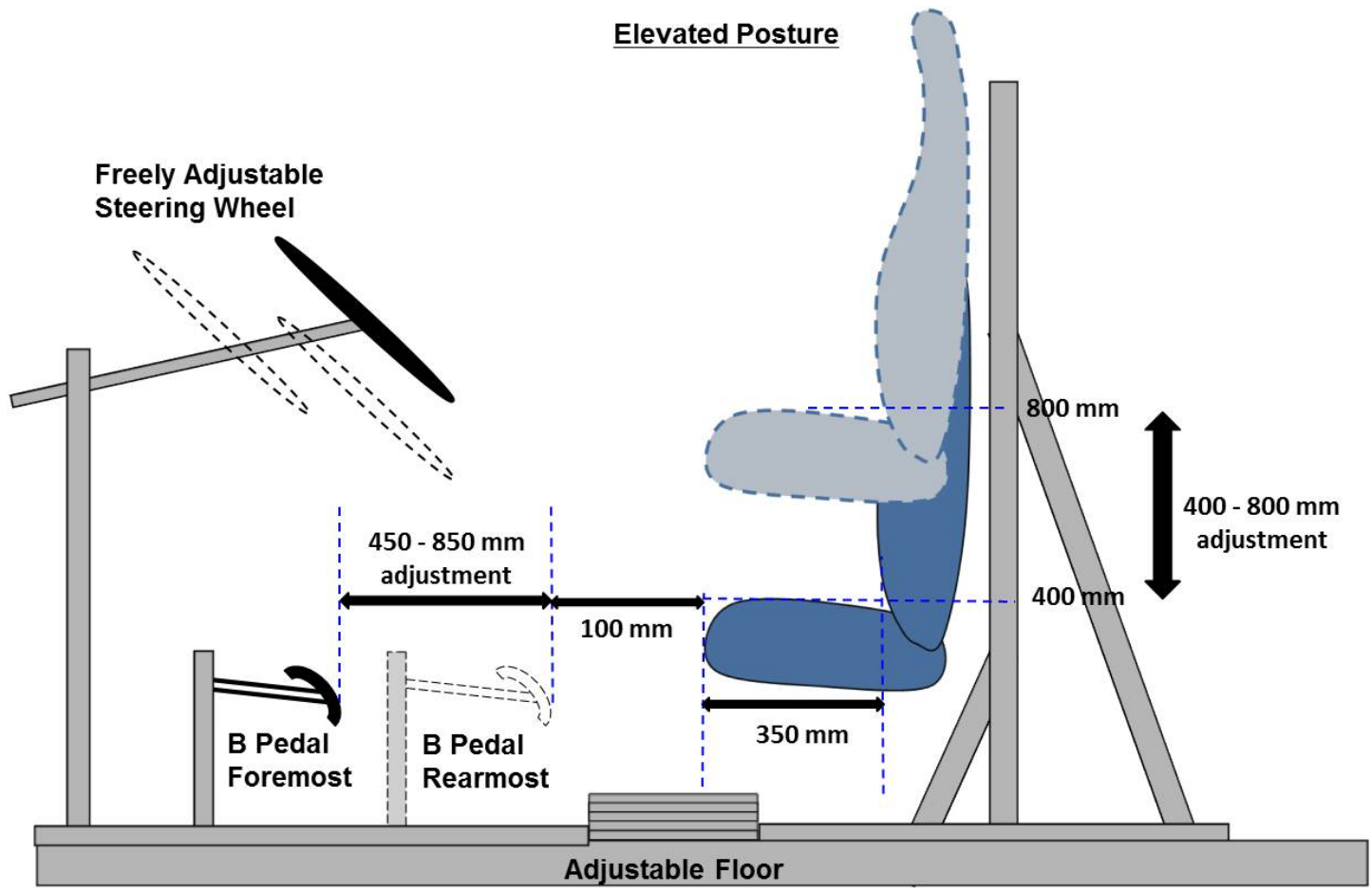

Figure 2. Image of seat and pedals used for the 'elevated posture' condition.

For the trial, each rig was mounted on to a Multi Axis Vibration Simulator (MAViS) at Loughborough University. The simulator has non-simultaneous excursions of $323 \mathrm{~mm}$ movement in X; $292 \mathrm{~mm}$ movement in Y; $184 \mathrm{~mm}$ movement in Z; 34 degrees roll; 34 degrees pitch; 52 degrees yaw. The simulator was set to use a pre-recorded reproduction of a pavé surface with a seat point vibration total value magnitude of $0.35 \mathrm{~m} / \mathrm{s}^{2}$ r.m.s. (weighted r.s.s. of $x-y$ - and z-axis motion). The vibration at the seat surface and platform was measured for each participant using a standard pad-mounted accelerometer on the seat surface and accelerometers mounted on the motion platform. The system settings were adjusted to compensate for the dynamics of the seat-person system so that the seat surface vibration was always set at the target level. Once the simulator was calibrated the pad was removed so to not influence discomfort ratings; the vibration at the seat base was kept constant and monitored throughout.

The driving simulation software (XPI Simulation) allowed both steering and pedal inputs and provided visual (Samsung 50" 1080p Screen) and audio outputs (Mackie Thump powered loudspeakers) for the driver. The software recorded and logged the performance over each scenario detailing various 
parameters. This included accelerator, brake and steering input (0-1 representing 'no input' to 'fully depressed/rotated') as well as the $x$ and $y$ coordinates of the centre of the driver's vehicle and other road occupying vehicles in the simulation. The visual field for the driver represented that through the front windscreen of a vehicle, with the speedometer located at the bottom (Figure 3). A rearview mirror was represented for the driver however wing mirrors were absent, due to the limited field of view, and as a result drivers were advised to use the rearview mirror and judge accordingly when changing lanes. Two screen positions were set (one for each trial) to address the difference in driver eye height between the standard and elevated driving posture. Having two heights ensured that drivers eye level matched up with the top third of the screen for both trials. With the one screen simulation system, the minor differences in eye level (based on anthropometry and seat height) did not adversely affect the visual feedback being presented on the screen.

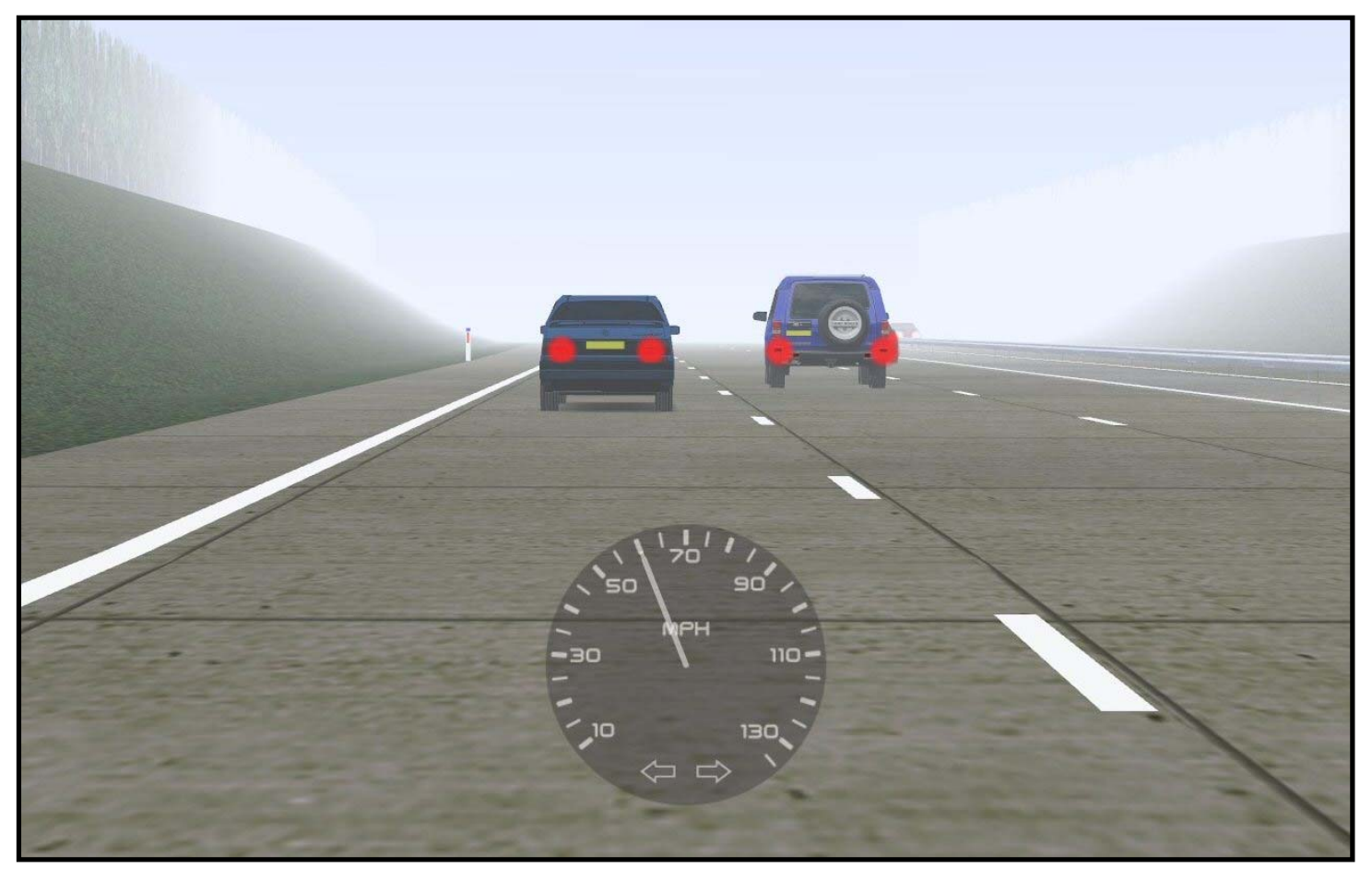

Figure 3. Screenshot of typical view in driving simulation - 'highway with fog'

\subsection{Driving Trial}

The driving simulation task was to follow a lead car at a safe distance (a distance which driver's would judge to be safe, feel comfortable with and self-select in road driving conditions), whose movement 
included changes of direction, speed and lane appropriate for normal driving. The driving trial consisted of six 10-minute scenarios, which included both town and highway driving conditions. In addition each trial included an adverse weather condition (fog) and also an emergency stop situation for one of the town and one of the highway driving scenarios. To control for learning effects, the order of testing was designed so that an identical highway scenario was tested for the first and last 10 minutes of the trial, for each participant, with a randomised order for the 4 scenarios in-between. Between each driving scenario, drivers remained seated for a few minutes whilst they were asked to rate their discomfort. Participants completed the trials for both driving postures. Half of the participants ( 3 males and 3 females) completed the elevated posture driving trial first and the other 6 participants completed the standard posture trial first. There was a 7-day gap between the first and second driving trial for each participant to mitigate learning effects between the two driving postures.

Before the driving trial, a short fitting trial was conducted with each participant, based on methods used by Porter and Gyi (2002) and Jones (1969), to obtain the preferred driving position for the seat and steering wheel position for both driving postures. The fitting trial was an iterative process based on the adjustment of components of the driving rig. For the new driving posture, the first component was the seat height, which was initially set at $60 \%$ of each driver's leg length. Each participant sat on the seat and set the height to their optimum comfort level, which was defined as the position where comfort could not be improved with further adjustment of the seat height. The distance from the seat to the pedals was determined by adjustment of the pedals in the fore-aft direction. The pedals began in the position furthest away from the seat and were brought towards the driver on a fully adjustable and lockable slide to the preferred position. The driver was asked to operate the pedals to obtain a feel for the optimum location. The third component for adjustment was the steering wheel position, which started in its furthest point away from the driver. The steering wheel had adjustment in overall height, distance from the driver and also the angle of the wheel itself. The driver was asked to take hold of the steering wheel and move it to their optimum position, where it was then locked into place. After all three components had been adjusted, the driver was asked if any or all of them needed further adjustment to improve comfort. If they did, the process was followed once more until the driver was satisfied with their settings. 
For the standard posture trial, the seat height and the steering wheel dimensions and adjustment ranges were fixed to the specification of the small LCV benchmark vehicle. The seat position, relative to the pedals, was adjusted on a pre-set adjustment slide to the participants preferred position in relation to the pedal set, replicating actual seat slide adjustment. The participant was asked to confirm once more that the seat position was in the best place for their comfort.

For each condition, once set in their preferred position, each participant was given 5 minutes on a Highway driving simulation scenario to familiarise themselves with the motion of the platform and the driving simulation software. After this trial run, participants were given a further opportunity to adjust their final driving position (before the trial began) to ensure that the ideal position was selected.

\subsubsection{Discomfort}

At the beginning of the first driving scenario and after each of the six subsequent 10-minute scenarios, each participant completed a body discomfort assessment, based on that used by Porter and Gyi (2002). The assessment collected data on 13 body parts on a 6-point comfort scale (1 not uncomfortable; 2 slightly uncomfortable; 3 fairly uncomfortable; 4 uncomfortable; 5 very uncomfortable; 6 extremely uncomfortable). The assessments were administered once the driving scenario was completed and the motion platform was no longer in motion. The scale verbal anchors were taken from ISO 2631-1 (Figure 4). 

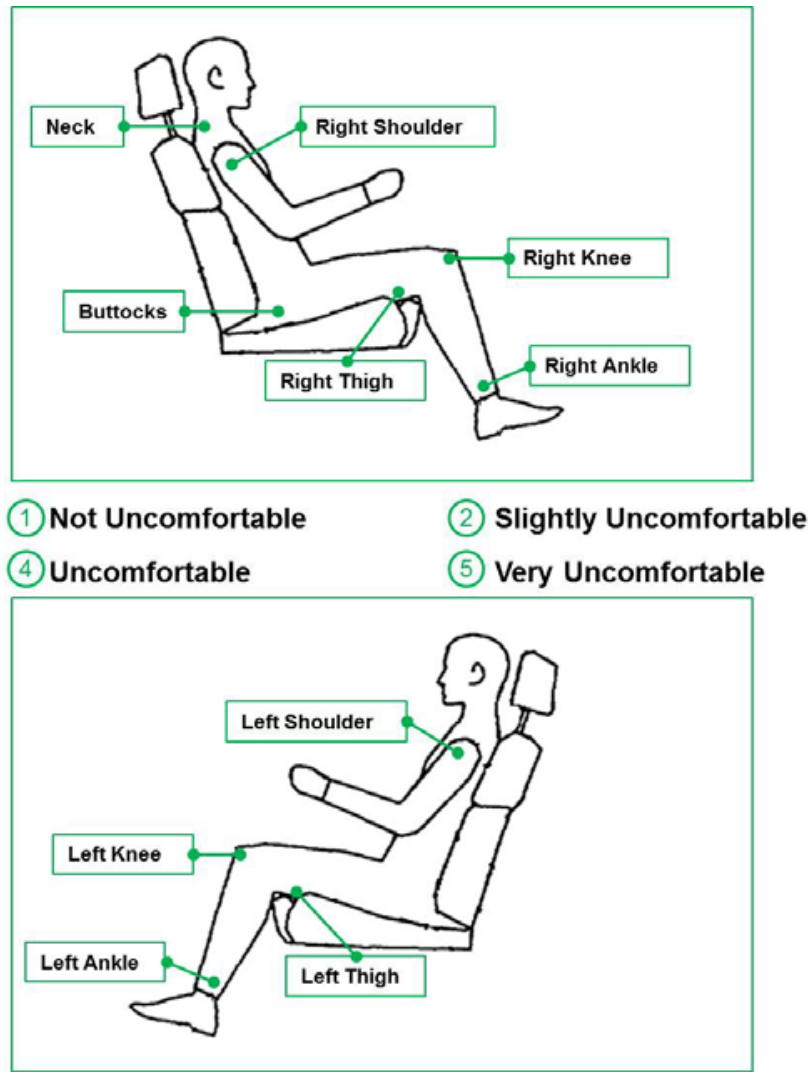

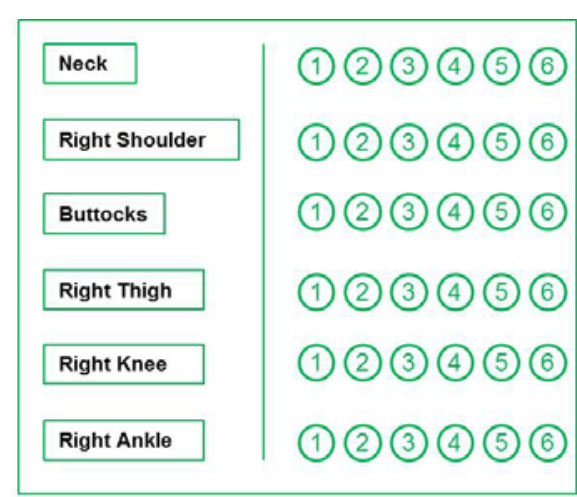

(3) Fairly Uncomfortable

(6) Extremely Uncomfortable
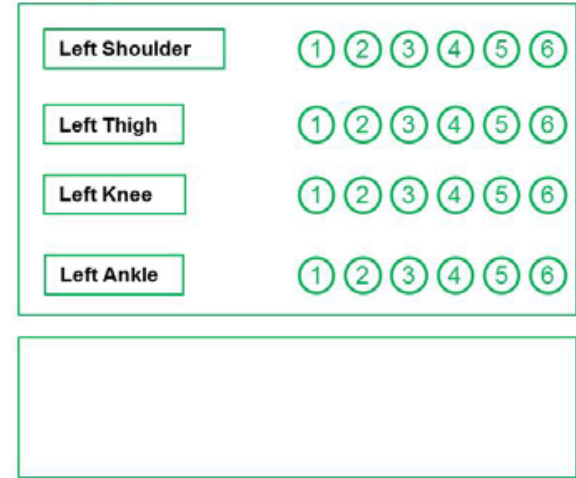

Figure 4. Body map used for subjective ratings of discomfort, and verbal anchors.

\subsubsection{Driver Performance}

The driving simulator outputs that were chosen to be used for this study were so based on the ease of incorporating them in to a real driving simulation, replicating manoeuvers which drivers might do on a day to day basis. The driving simulator logged driver and lead-car road position at 30 samples per second; this allowed calculation of continuous headway. The analysis used headway for the highway scenario, which was used for the first (0-10 minutes) and the last scenarios (50-60 minutes), for both driving postures. Besides the lead car, no traffic was present for the town driving scenarios and so the highway scenario was therefore chosen based on the presence of more road traffic, which was negotiated through lane changes and overtaking and presented a more realistic driving experience.

The literature suggests that an effective method of breaking down reaction time can be to split the action in to a number of steps, the key one being the transfer of the operating foot from the A pedal to the B pedal (Green, 2000; Engström et al, 2010). For this study, reaction time was defined as the time 
(seconds) taken from the emergency stop instruction, to initial contact with the brake pedal in response. This method was chosen to understand the effects of biomechanically moving the operating foot from the A pedal to the B pedal, if any, when sat in an elevated driving posture and immersed in a driving task. Pilot work showed that the simulator immersed drivers in the task effectively and so towards the end of a 10-minute driving scenario, their attention was very much on negotiating their driving around the route. This was found from pilot participants reporting mental fatigue from the driving task. The simulation system logged the accelerator and brake input across the 10-minutes (0-1) as well as the time at which the emergency stop command was issued. The data provided the time at which the accelerator pedal had no input (no depression of pedal) and the subsequent time at which the brake pedal had input (pedal depression) in hundredths of a second values. The emergency stop was presented to the driver both visually, with a large UK Highway Code standardised 'STOP' sign flashing on the screen and audibly, with a pre-recorded and synchronized voice instructing the driver to stop. This occurred during two of the driving scenarios (one town and one highway). Before the trial, participants were briefed that they would encounter an emergency stop situation and the nature of this instruction, (to brake hard until the vehicle came to a complete stop); however they were not briefed on exactly when this would be. The emergency stop instruction triggered at 9.5 minutes in to the driving scenarios and so it was impossible to know exactly where drivers would be on the route when this occurred and thus whether they were using the accelerator or brake pedal at this time. The mitigation for this was having this feature in highway driving as it is a constant piece of road where speed can be managed for the most part by managing the compression of the accelerator. This helped to ensure that drivers would have at least one scenario where the reaction time was truly from the accelerator to the brake pedal.

\section{Results}

\subsection{Sampling}

Descriptive statistics for the sample are detailed in Table 1 (age/gender) and Tables 2 and 3 (anthropometry). The sample had an equal spread (6 males and 6 females) with an age range from 19-60, with a leg length range (crucial dimension in seat set up) from Japanese female 31st percentile (JF31) to American male 95th percentile (AM95). 
Table 1. Descriptive statistics from the participant sample $(n=12)$

\begin{tabular}{l|c|c}
\hline Participant & Age & Gender \\
\hline 1 & 23 & Male \\
2 & 19 & Female \\
3 & 32 & Male \\
4 & 25 & Female \\
5 & 39 & Male \\
6 & 31 & Female \\
7 & 30 & Male \\
8 & 60 & Female \\
9 & 50 & Male \\
10 & 57 & Female \\
11 & 44 & Male \\
12 & 36 & Female \\
\hline
\end{tabular}

Table 2. Anthropometric data from the male participant sample $(n=6)$

\begin{tabular}{l|c|c|c|c|c|c}
\hline Dimension (mm) & P1 & P3 & P5 & P7 & P9 & P11 \\
\hline Stature & 1820 & 1833 & 1824 & 1849 & 1723 & 1731 \\
Sitting Height & 942 & 958 & 953 & 967 & 906 & 906 \\
Shoulder Width & 437 & 419 & 428 & 453 & 443 & 424 \\
Sitting Hip Width & 391 & 369 & 458 & 414 & 449 & 406 \\
Knee Height & 548 & 564 & 554 & 535 & 514 & 522 \\
Popliteal Length & 479 & 488 & 520 & 493 & 500 & 505 \\
Seat Height & 440 & 417 & 416 & 430 & 390 & 440 \\
Leg Length & 1040 & 1129 & 1066 & 1157 & 1027 & 984 \\
Foot Length & 280 & 275 & 270 & 265 & 265 & 260 \\
\hline
\end{tabular}


Table 3. Anthropometric data from the female participant sample $(n=6)$

\begin{tabular}{l|c|c|c|c|c|c}
\hline Dimension (mm) & P2 & P4 & P6 & P8 & P10 & P12 \\
\hline Stature & 1600 & 1645 & 1700 & 1682 & 1639 & 1680 \\
Sitting Height & 870 & 880 & 920 & 851 & 876 & 874 \\
Shoulder Width & 400 & 370 & 380 & 389 & 382 & 375 \\
Sitting Hip Width & 381 & 373 & 337 & 335 & 399 & 429 \\
Knee Height & 492 & 515 & 521 & 503 & 490 & 502 \\
Popliteal Length & 470 & 470 & 450 & 492 & 448 & 476 \\
Seat Height & 390 & 430 & 450 & 401 & 375 & 380 \\
Leg Length & 883 & 901 & 967 & 975 & 947 & 939 \\
Foot Length & 220 & 230 & 260 & 250 & 230 & 235 \\
\hline
\end{tabular}

\subsection{Discomfort}

The body discomfort assessments provided 7 discomfort ratings for each participant, for each of the 13 body parts. All comparisons used the paired-samples t-test technique, chosen as a parametric test for 1 sample, under two different conditions (standard and elevated posture).

For the elevated posture, there was a significant increase in discomfort ratings between the first $(0$ minutes) and last (60 minutes) for the neck, $t(11)=-3.63, p<.005$ (mean change from 1.2 to 2.3 ); left shoulder, $t(11)=-2.55, p<.05$ (1.2 to 1.8$)$; upper back, $t(11)=-3.45, p<.005(1.3$ to 2.0$)$ and right ankle, $t(11)=-3.95, p<.005$ (1.3 to 2.8$)$. The neck, upper back and right ankle for the elevated posture increased by at least one increment on the scale, indicating that these body parts were affected most after 60-minutes. For the standard posture there was a significant increase in discomfort in the left shoulder, $t(11)=-2.57, p<.05$ (mean change from 1.1 to 1.6 ); right shoulder $, t(11)=-2.45, p<.05(1.1$ to 1.8 ); upper back, $t(11)=-3.45, p<.005$ (1.1 to 1.8$)$; middle back, $t(11)=-2.60, p<.05$ (1.0 to 1.7$)$ and buttocks, $t(11)=-2.35, p<.05(1.0$ to 1.3$)$. 
Results showed that differences in discomfort observed between the standard and elevated posture at the end of the trial (60 minutes) were significant only for the right ankle $(t(11)=3.98, p<.005)$ with the elevated posture having the higher discomfort ratings (Figure 5).

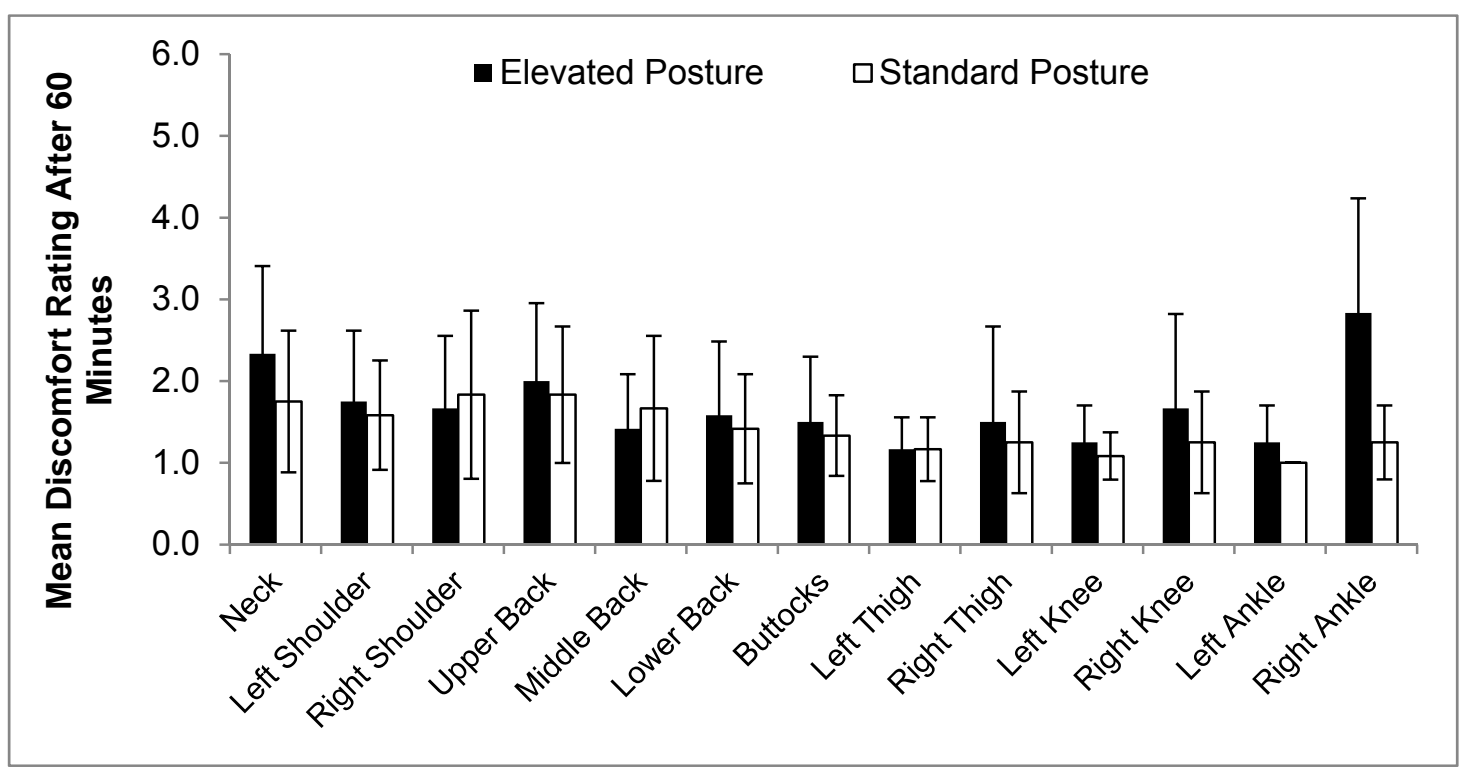

Figure 5. Discomfort ratings measured at 60 minutes for the two postures (mean and standard deviation).

\subsection{Driver Performance}

The driver performance was measured in terms of both 'time headway', defined as the time it would take the driven vehicle at its current speed to travel the distance from the centre of the driven vehicle to the centre of the lead vehicle and the 'reaction time', defined as the time taken from the emergency stop visual trigger to the operation of the brake pedal. This data was extracted and analysed using a one-way repeated measures ANOVA test.

\subsubsection{Headway}

For the standard posture, the mean 'time headway' reduced from 2.0 seconds to 1.7 seconds over the duration of the trial. By the end of the trial (60 minutes), the mean headway for the elevated posture was 1.5 seconds, compared with the standard posture of 1.7 seconds (Figure 6). Differences between 
headway at 60 minutes were significant for the elevated posture, showing a shorter headway compared with the first 10 minutes of driving $(F(1,11)=5.17, p<.05)$.

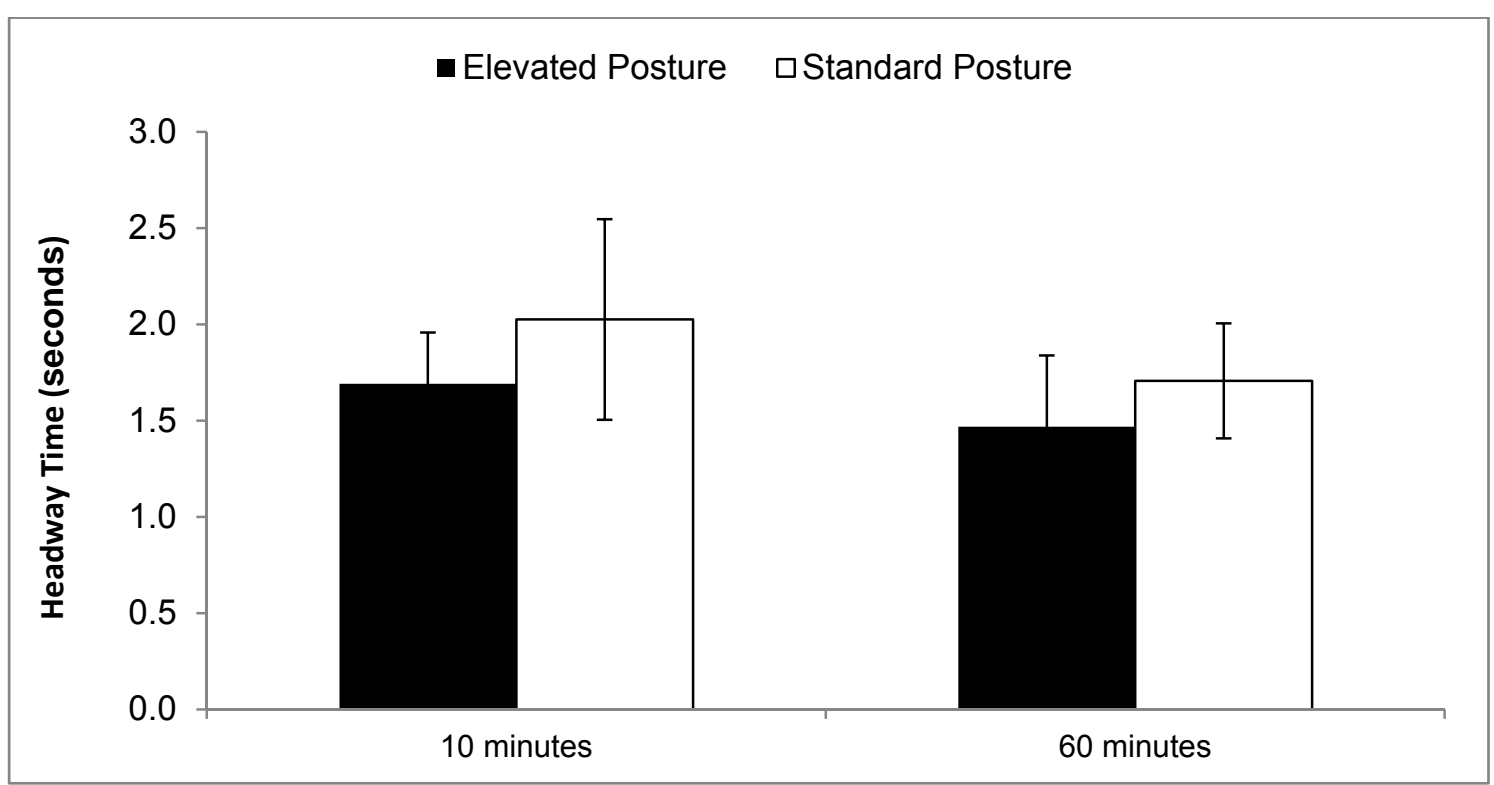

Figure 6. Comparison of headway (seconds) for first scenario (10 minutes) and final scenario (60 minutes) for the elevated and standard posture (mean and standard deviation).

\subsubsection{Reaction Time}

Mean reaction time was slightly shorter for the elevated posture in both the highway and town driving scenarios, although these differences were not significant (Figure 7). In the highway driving, the mean reaction time for the elevated posture was 0.82 seconds, compared with 0.84 seconds for the standard posture. In the town driving, the mean reaction time for the elevated posture was 0.75 seconds, compared with 0.94 seconds for the standard posture. 


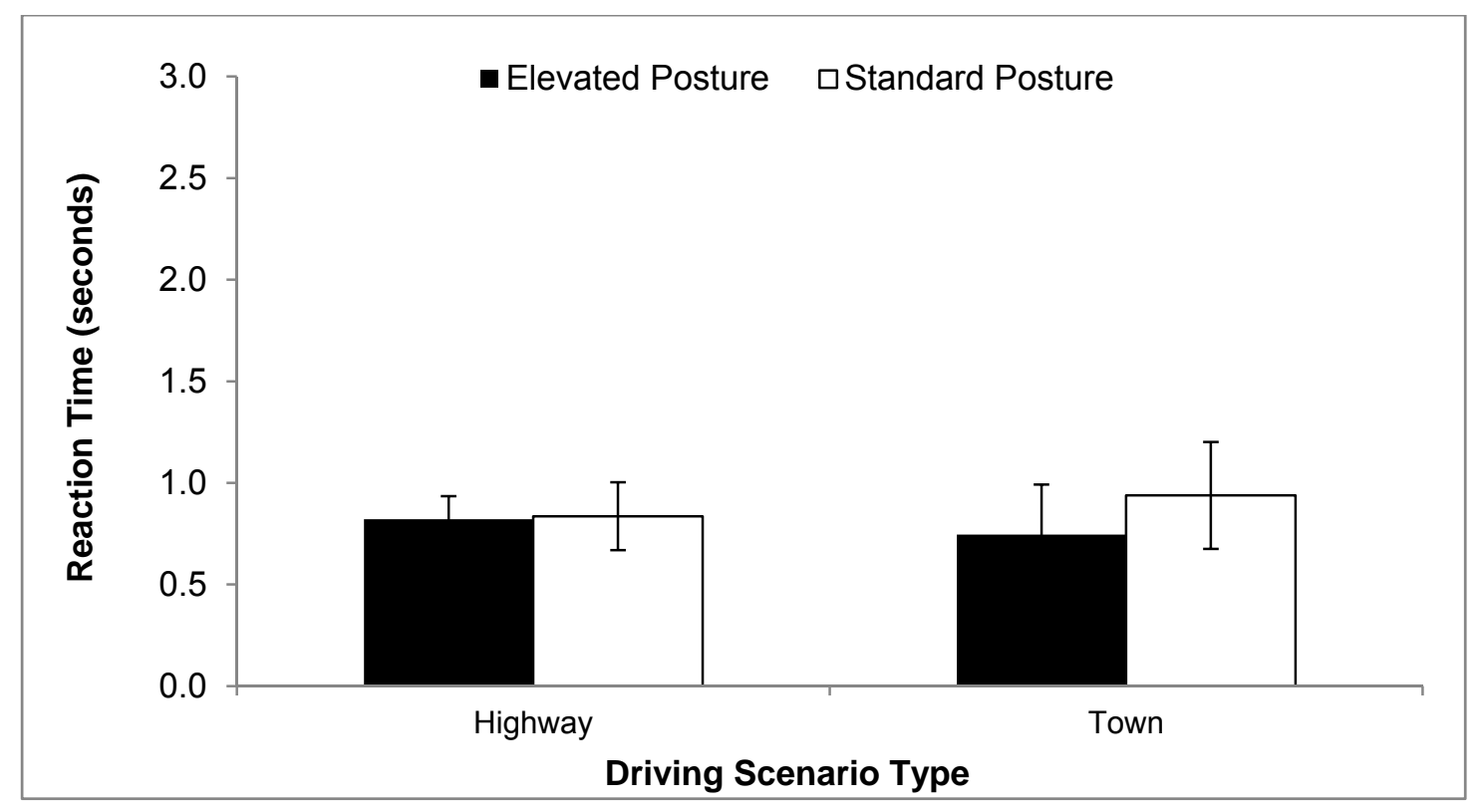

Figure 7. Comparison of reaction time (seconds) for emergency stop in the highway and town scenarios for the elevated and standard posture (mean and standard deviation).

\section{Verification Trial}

A key finding from the main trial was that there was a significant difference in discomfort for the right ankle between the standard and elevated driving postures. It was identified that there was a difference between the two driving rigs relating to the force needed to operate the pedals, with more pedal stiffness for the standard posture right $(40 \mathrm{~N})$ compared with that of the elevated posture rig $(20 N)$. As a result it was important to explore this further by addressing the stiffness of the pedals and matching them for both rigs in a verification trial. Comparisons were made using the independentsamples t-test technique, chosen as a parametric test for 2 samples, under the same conditions.

A trial was designed for an independent sample of 12 participants, 6 males and 6 females, and was completed in the elevated posture only. The task of the driving simulation was to follow the lead car in front and replicate the movement and maneuverability of that car, following the same experimental design as the main trial. The driving trial consisted of five 10-minute scenarios, which included both town and highway driving conditions and was 10 minutes (1 scenario) shorter in length than the previous trial. The main trial showed that significant differences in discomfort fatigue effects were observed after 50 minutes of driving, indicating that 50 minutes was sufficient time to collect adequate ankle discomfort data. The trial still included an adverse weather condition (fog) and also an 
emergency stop situation on both the town and highway driving scenarios. To control for learning effects, the order of testing was designed so that the identical highway scenario was tested for the first and last 10 minutes of the trial, for each participant, with a randomised order for the 3 scenarios in-between. The Mann-Whitney test technique was used to analyse the data, as a non-parametric equivalent of the paired t-test for independent samples (12 participants from main trial and 12 independent participants for the verification).

The pedal force (i.e. spring stiffness) was increased from $20 \mathrm{~N}$ to $40 \mathrm{~N}$ to meet that of the standard posture driving rig in the main trial, which itself was set to replicate the benchmark production vehicle. Figure 8 shows the right ankle discomfort scores recorded at the beginning ( 0 minutes) and end of the trial (50 minutes) were comparable with those recorded in the first trial.

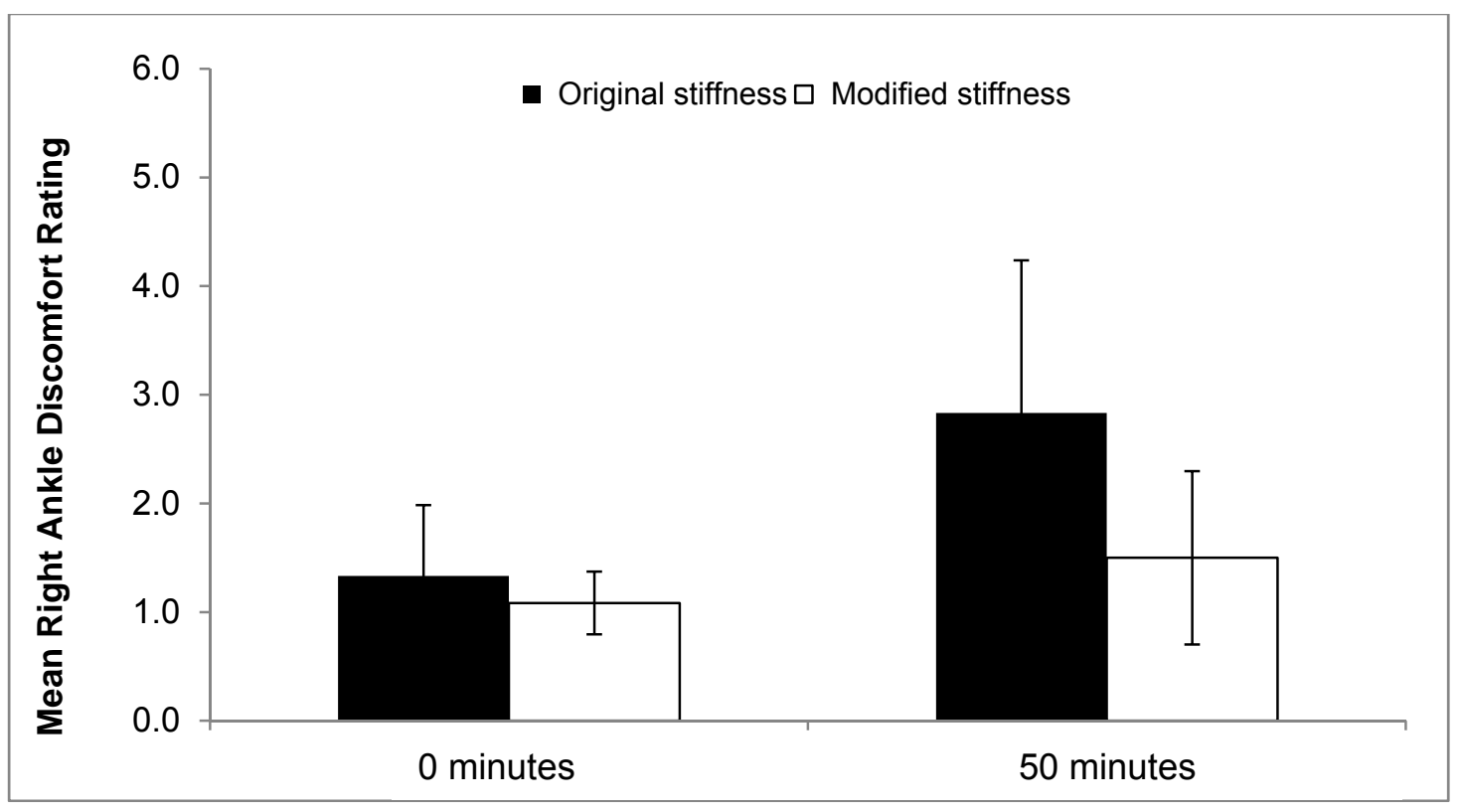

Figure 8. Comparison of discomfort ratings for the right ankle measured at 0 and 50 minutes for the original (soft) pedal and modified (firm) pedal stiffness (mean and standard deviation).

For both trials the right ankle discomfort increased between the first ( 0 minutes) and last (60 minutes and 50 minutes respectively) mean discomfort score. There were significant differences in the right ankle discomfort data between the main trial $(M=2.83, S D=1.40)$ and the verification trial $(M=1.50$, $S D=0.80) ; t(17.44)=2.86, p=.01$ (two-tailed) with 9 out of 12 participants rating the ankle 
discomfort lower for the verification trial with 3 tied ratings. The results from the verification trial therefore indicate that low pedal force and the consequent lack of support for the foot was likely to be the cause of the ankle discomfort.

\section{Discussion}

\subsection{Discomfort}

The results indicate that the elevated posture showed fewer statistically significant increases in discomfort after 60 minutes of driving, in comparison with the standard posture. However, the magnitude of the discomfort changes for each body zone was similar for both postures, with the exception of the right ankle. The right ankle showed a higher level of discomfort for the elevated posture, which was explained and explored with the verification trial. The fundamental design of the elevated posture, with the angle from which the legs interact with the pedals becoming steeper, means that the seat length needs to be shorter to ensure easier maneuverability of the legs for pedal operation. With this design, the weight of the occupant primarily shifts towards the front edge of the seat (beneath the thighs) as opposed to the weight distribution being at the back of the seat surface (beneath the buttocks of the occupant) in a conventional set up. Although several participants raised concerns over the shorter seat base length for the elevated posture, the fatigue effects indicated that there was no significant increase in buttock discomfort when compared to the standard posture. This finding indicates that the shorter seat base length had no detrimental effects to the occupant seating comfort for this one-hour trial, despite the weight distribution being shifted forward for the occupant. In a conventional seated posture, the curve of the lumbar spine is flattened and changes shape from that of a standing posture with a healthy ' $S$ ' shape spine. This increases pressure in the posterior part of the inter-vertebral discs and within the nucleus itself, making it vulnerable to long-term damage. The elevated posture packages occupants in a more upright position in the seat, keeping the back closer to the 'S' shape as observed in standing. The results of this study showed that whilst there was no significant changes in discomfort for the elevated posture below the upper back, for the standard posture there were significant changes in discomfort across the trial for both the middle back and the buttocks. This suggests that over time the lower portion of the back and buttocks are affected more by discomfort in driving, and that the elevated posture could go some way to relieving this commonly experienced problem. 
Every body part, for both postures, had an increased discomfort score between 0 and 60 minutes, indicating that fatigue effects occurred and fell in line with the expectation for long duration testing (Griffin et al, 1982; Gyi and Porter, 1999; El Falou et al, 2002).

\subsection{Driver Performance}

\subsubsection{Headway}

This study shows that drivers had a consistently shorter headway across the duration of the 60minute trial, for both postures. These findings support those of Van Winsum (1996) who concluded that when drivers were able to judge the intensity of braking levels, they drove with consistently shorter headways. They also observed that both driving behavior and physical limitations contribute to selfselecting an appropriate headway. This finding also indicates adaptation of the driver, possibly due to increasing familiarity and confidence with the driving simulator. Participants reported that being higher on the road in the elevated posture gave a greater feeling of control when driving and this could explain the increased confidence being reflected in a reduced headway in comparison to the standard posture.

The mean self-selected headway by the end of the driving trial was 1.7 seconds and 1.5 seconds for the standard posture and the elevated posture, respectively. The Highway Code (Department for Transport, 2007) guidance advises drivers to allow at least a two-second gap between them and the vehicle in front, which is considered the minimum safe distance. Lee (1976), in a study of braking based on information about time-to-collision, stated that the safe time headway is dependent on various factors and uses 2-seconds as a reference. However, Treiterer and Nemeth (1970) and von Buseck et al (1980) reported actual highway headways (based on road data) of between 1 and 2 seconds, which gives a much more lenient range on the guidance for safe headway and corresponds with the self-selected headways observed from this study. Furthermore, Simons-Morton et al (2005) classify 'risky' driving as headways of $<1.0$ seconds, whereas Rudin-Brown et al (2004) defined headway as being too short at $<1.5$ seconds, which are both shorter than the mean headway 
observed at the end of driving. The literature led to a safe 'time headway' classification of $\geq 1.5$ seconds in normal driving conditions, which was observed in this study.

Whilst minimising discomfort has become a fundamental concern in the development of car seats, safety remains of paramount importance when packaging a driver. As an unexplored driving posture, with a change in the fundamental mechanics of sitting, it is essential to understand how drivers physically interact with the environment around them in this elevated posture. This finding indicates that drivers maintain the ability to control the vehicle safely whilst packaged in an elevated driving posture, which is comparable to both the literature and to the results observed of those same drivers in a conventional set-up.

\subsubsection{Reaction Time}

These reaction times are comparable to those observed for the accelerator-brake reaction time in the study by Engström et al. (2010), who showed that the accelerator-brake reaction times observed were between $0.6-0.8$ seconds. Although there is no evidence that the elevated posture increases the reaction time, the trend of a shorter reaction time for the elevated posture could indicate that it is easier to switch operation between the A and B pedal during driving, compared with the standard posture. This finding helps to understand the biomechanics of this posture further in terms of comfort, design and safety. As an unexplored posture, and with the knowledge that more of the occupant's weight is shifted towards the front edge of the seat and the feet, it was unknown as to whether conventional pedal operation could be utilised. These findings indicate that drivers can comfortably operate a conventional pedal set up (switching between pedals) and that they can do so safely (in a time comparable to the literature, comparable to drivers in the conventional driving posture and without any functional issues). For the design of this posture, this shows that changing the biomechanics of the driver resulting in a space saving in the vehicle is possible to do without adversely affecting the comfort and control of the driver. 


\section{Conclusions}

This study confirms that drivers were able to find a comfortable driving position for vehicle performance in the elevated posture $(>400 \mathrm{~mm})$. Drivers were subjected to a 60 -minute driving simulation, during which the levels of discomfort were comparable and not significantly worse than those experienced in the standard posture under the same conditions. In both postures drivers experienced fatigue effects throughout the duration of the trial and significant differences were observed in the upper body zones, especially in the back and shoulders with more discomfort reported at the end of the trials. Ankle pain was associated with the force (spring stiffness) in the accelerator pedal and was mitigated by appropriate pedal modification.

The results showed that the self selected headway for the elevated posture was comparable and not significantly different to that of the standard posture, whilst falling in line with safe distances as cited in the literature. The study showed that the reaction time for the elevated posture was comparable and not significantly different to that of the standard posture, and the accelerator-brake reaction times were comparable to those previously reported.

It is concluded that the elevated driving posture did not adversely affect the driver discomfort or performance for the tests conducted in this study.

\subsection{Limitations}

For the reaction time test, the emergency stop instruction triggered at 9.5 minutes in to the preprogrammed driving scenarios making it difficult to assume drivers were operating the A pedal at this point.. For the trial, this is why highway driving was chosen for this test. However, given that local driving often leads to more unexpected events than motorway driving, it would be beneficial to examine reaction time in town driving scenarios. For headway, highway driving scenarios were used. The limitation of the software programmed for this study was that besides the lead car, no traffic was present for the town driving scenarios and so it didn't reflect real world driving as much as the traffic incorporated highway scenarios did. 
The visual field of the driver changed from standard to elevated driving posture, an angle which was determined by their anthropometry and elevated posture seat height (self-selected). Two screen heights were set based on the driving posture (standard or elevated), judged to be a good fit for the sample of drivers used. However, the limitations of the equipment and the laboratory space meant that specific screen height adjustment could not be offered from driver to driver, which therefore made it difficult to ensure that the drivers' visual angles were identical between postures.

Studies have shown that whilst some seats can be considered uncomfortable after 15 minutes, others that were considered comfortable become uncomfortable after about one hour (Porter et al, 2003). Previous research has also implemented a range of trial durations from 60-seconds to 135 minutes (Kolich, 2003a; Gyi and Porter, 1999) with findings demonstrating that trial duration (temporal factors) greatly influence driver' overall discomfort, with significant changes in discomfort occurring at approximately 80 to 110 minutes of driving (Gyi and Porter 1999). This study was designed for a 60minute exposure to the driving task with vibration, limited by time restraints and participant availability. More long-term trials would be beneficial to understand the temporal effects further under driving in an elevated posture.

\section{References}

DEPARTMENT FOR TRANSPORT, 2007. Highway Code. HMSO, ISBN 0115528148.

EL FALOU, W. et al., 2002. Evaluation of driver discomfort during long-duration car driving. Applied Ergonomics, 34(3), 249 - 255.

ENGSTRÖM, J. et al., 2010. Effects of working memory load and repeated scenario exposure on emergency braking performance. Human Factors: The Journal of the Human Factors and Ergonomics Society, 52, 551.

GREEN, M., 2000. How long does it take to stop? A methodological analysis of driver perceptionbrake times. Transportation Human Factors, 195 - 216.

GRIFFIN, M. et al., 1982. Vibration and comfort. I: Translational seat vibration. Ergonomics, 25, 603 630.

GYI, D.E. and PORTER, J.M., 1999. Interface pressure and the prediction of car seat discomfort. Applied Ergonomics, 30(2), 99 - 107.

GYI, D.E. and PORTER, J.M., 1999. Interface pressure and the prediction of car seat discomfort. Applied Ergonomics, 30(2), 99 - 107. 
JONAH, B.A., 1996. Sensation seeking and risky driving: a review and synthesis of the literature. Road Safety Directorate, Transport Canada.

JONES, J.C., 1969. Methods and results of seating research. Ergonomics, 12(2), 171 - 181.

KOLICH, M., 2003. Automobile seat comfort: occupant preferences vs. anthropometric accommodation. Applied Ergonomic, 34(2), 177 - 184.

LEE, D.N., 1976. A theory of visual control of braking based on information about time-to-collision. Perception, 5, 437 - 459.

MANSFIELD, N.J., MACKRILL, J., RIMELL, A.N., and MACMULL, S.J. (2014). Combined effects of long-term sitting and whole-body vibration on discomfort onset for vehicle occupants. ISRN Automotive Engineering, 2014, 1-8.

MANSFIELD, N.J., 2005. Human Response to Vibration, CRC press.

PORTER, J.M. and GYI, D.E., 1998. Exploring the optimum posture for driver comfort. Int. J. of Vehicle Design, 19(3), 255 - 266.

PORTER, J.M. and GYI, D.E., 2002. The prevalence of musculoskeletal troubles among car drivers. Occupational Medicine, 52(1), 4 - 12.

PORTER, J.M., GYI, D.E., and TAIT, H.A., 2003. Interface pressure data and the prediction of driver discomfort in road trials. Applied Ergonomics, 34(3), 207 - 214.

REBIFFE, R., 1969. The driving seat: Its adaption to functional and anthropometric requirements. Proceedings of a Symposium on Sitting Posture, 132 - 147.

RUDIN-BROWN, C.M., and PARKER, H.A., 2004. Behavioural adaptation to adaptive cruise control (ACC): implications for preventative strategies. Transportation Research Part F7: Traffic Psychology and Behaviour, 7(2). 59 - 76.

SIMONS-MORTON, B. et al., 2005. The observed effects of teenage passengers on the risky driving behaviour of teenage drivers. Accident Analysis and Prevention, 37(6), 973 - 982.

TREITERER, J., and NEMETH, Z.A., 1970. Multiple rear-end collisions in freeway traffic, their causes and their avoidance. Society of Automotive Engineers, SAE 700085.

VAN WINSUM, W. and HEINO, A., 1996. Choice of time headway in car-following and the role of time-to collision information and braking. Ergonomics, 39, 579 - 592.

VON BUSECK, C.R. et al., 1980. Seat belt usage and risk taking driving behaviour. Presented at the Society of Automotive Engineers Congress. 